Supporting Information for

\title{
A Versatile Strategy to Coat Individual Cell with Fully/Partially Covered Shell for Preparation of Self-Propelling Living Cells
}

Chong Jiao, ${ }^{a, b}$ Changwen Zhao, ${ }^{*}, a, b, c$ Yuhong Ma, ${ }^{d}$ Wantai Yang ${ }^{*}, a, b, c, d$

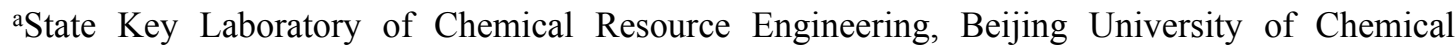
Technology, Beijing, 100029, China

${ }^{b}$ Beijing Laboratory of Biomedical Materials, Beijing University of Chemical Technology, Beijing, 100029, China

${ }^{c}$ Key Laboratory of Biomedical Materials of Natural Macromolecules, Ministry of Education Beijing, Beijing University of Chemical Technology, 100029, China

${ }^{\mathrm{d}}$ Key Laboratory of Carbon Fiber and Functional Polymers Ministry of Education, Beijing University of Chemical Technology, Beijing, 100029, China

E-mail: zhaocw@mail.buct.edu.cn (C. Z.); yangwt@mail.buct.edu.cn (W. Y.) 


\section{Table of Contents}

Figure S1. Micrographs of yeast cells adsorbed with PEI deposited on the surface of the slide.

Figure S2. FTIR spectra of (a) native yeast cells, (b) yeast cells adsorbed with PEI, (c) poly(PEGDA) and (d) cell@poly(PEGDA).

Figure S3. CLSM images of cell@poly(PEGDA) prepared at $5 \mathrm{~mW} / \mathrm{cm}^{2}$ for 20, 40, 60 $\min$.

Figure S4. CLSM images of cell@poly(PEGDA) prepared at $10 \mathrm{~mW} / \mathrm{cm}^{2}$ for 20, 40, $60 \mathrm{~min}$.

Figure S5. (a, a1, a2) CLSM images of Janus-poly(PEGDA)-cell without Bio-PEGACA after fluorescent staining. (b, b1, b2) CLSM images of Janus-poly(PEGDA)-cell without copolymerization of Bio-PEG-ACA with AAS after fluorescent staining.

Video S1. Propulsion of Janus-Urease-cell in the presence of $0 \mathrm{mM}$ urea.

Video S2. Propulsion of Janus-Urease-cell in the presence of $50 \mathrm{mM}$ urea.

Video S3. Propulsion of Janus-Urease-cell in the presence of $100 \mathrm{mM}$ urea.

Video S4. Propulsion of Janus-Urease-cell in the presence of $200 \mathrm{mM}$ urea. 


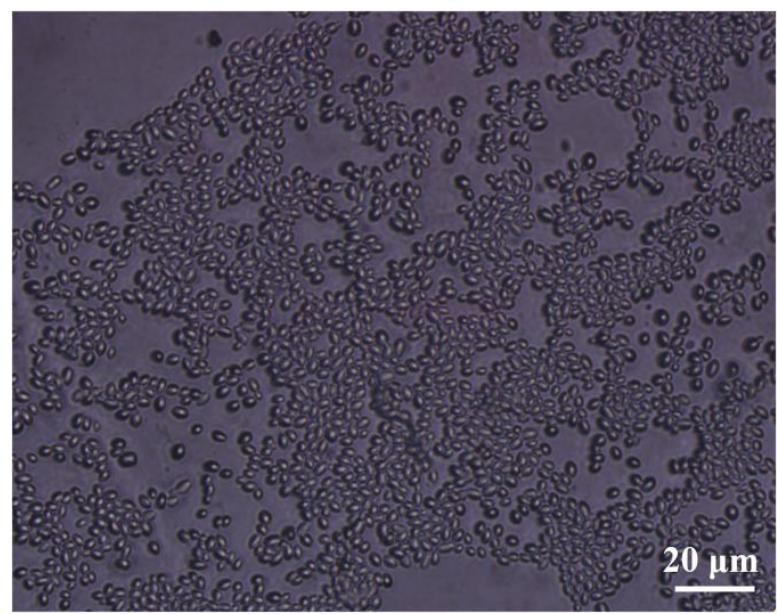

Figure S1. Micrographs of yeast cells adsorbed with PEI deposited on the surface of the slide.

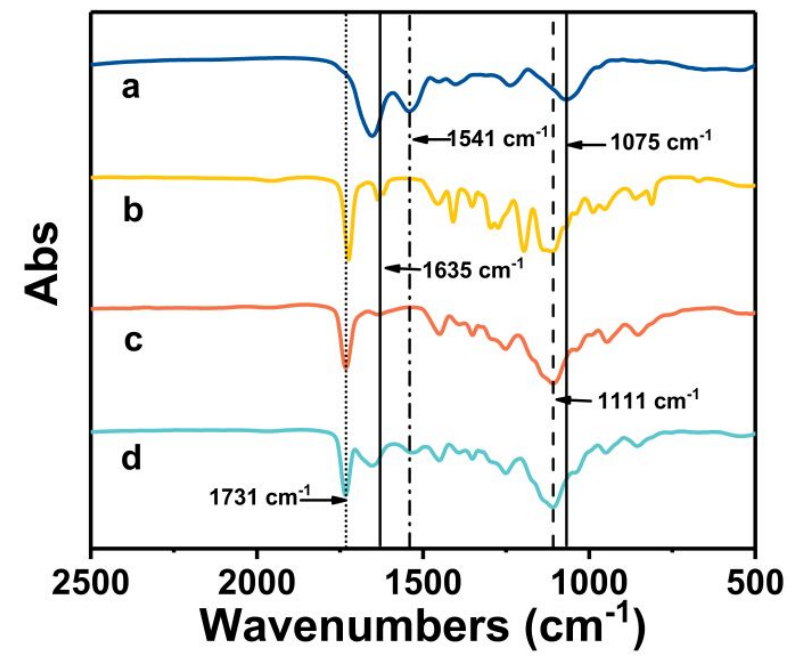

Figure S2. FTIR spectra of (a) native yeast cells, (b) yeast cells adsorbed with PEI, (c) poly(PEGDA) and (d) cell@poly(PEGDA). 


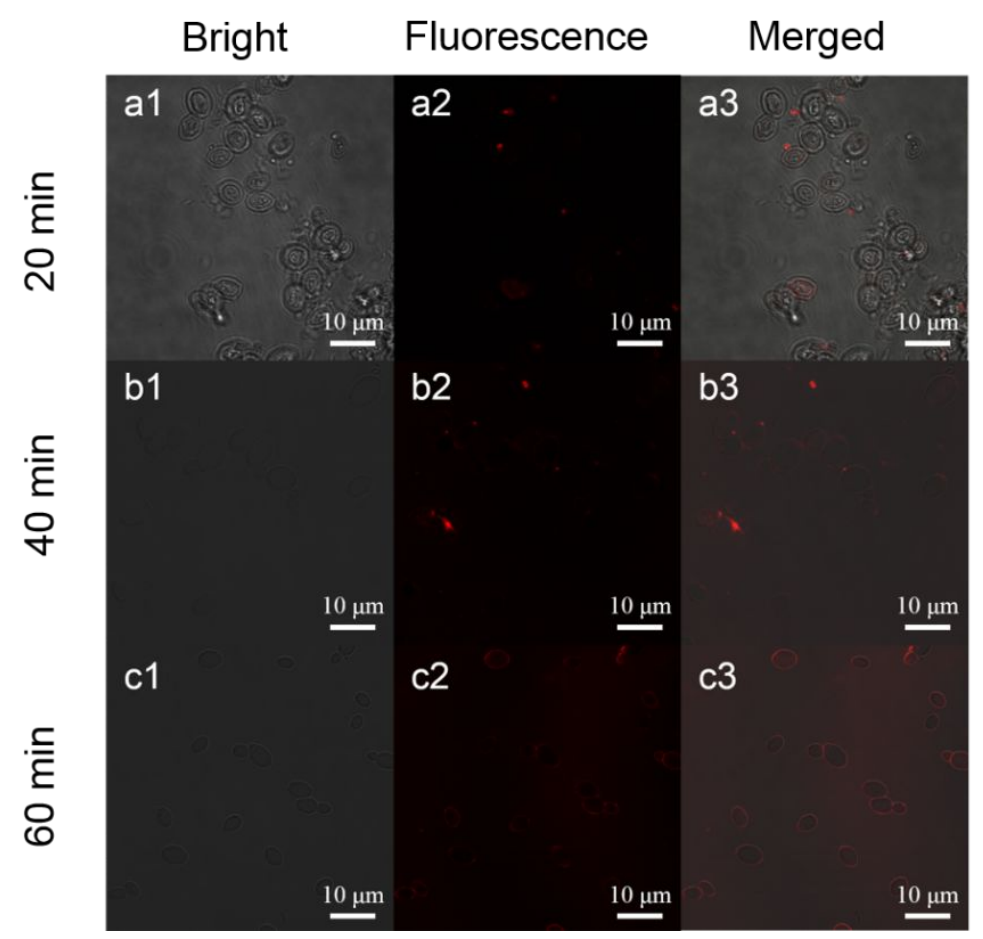

Figure S3. CLSM images of cell@poly(PEGDA) prepared at $5 \mathrm{~mW} / \mathrm{cm}^{2}$ for 20, 40, 60 min.

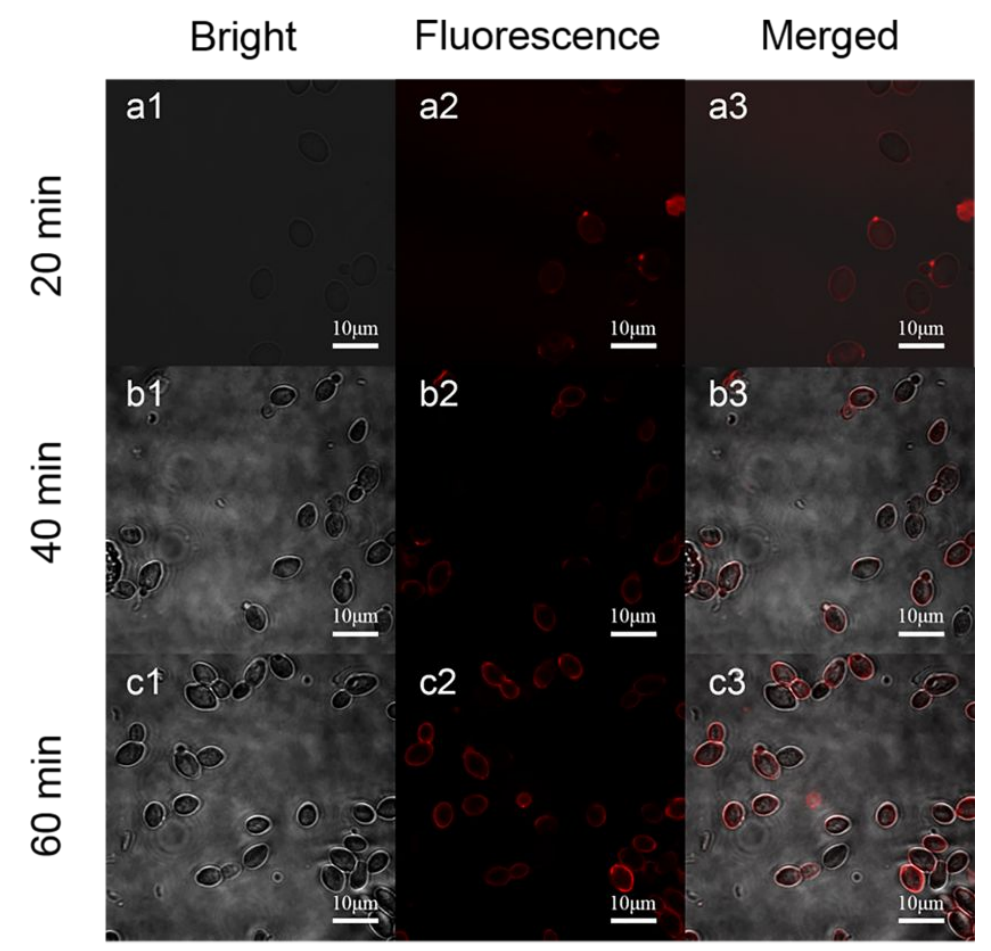

Figure S4. CLSM images of cell@poly(PEGDA) prepared at $10 \mathrm{~mW} / \mathrm{cm}^{2}$ for 20, 40, $60 \mathrm{~min}$. 


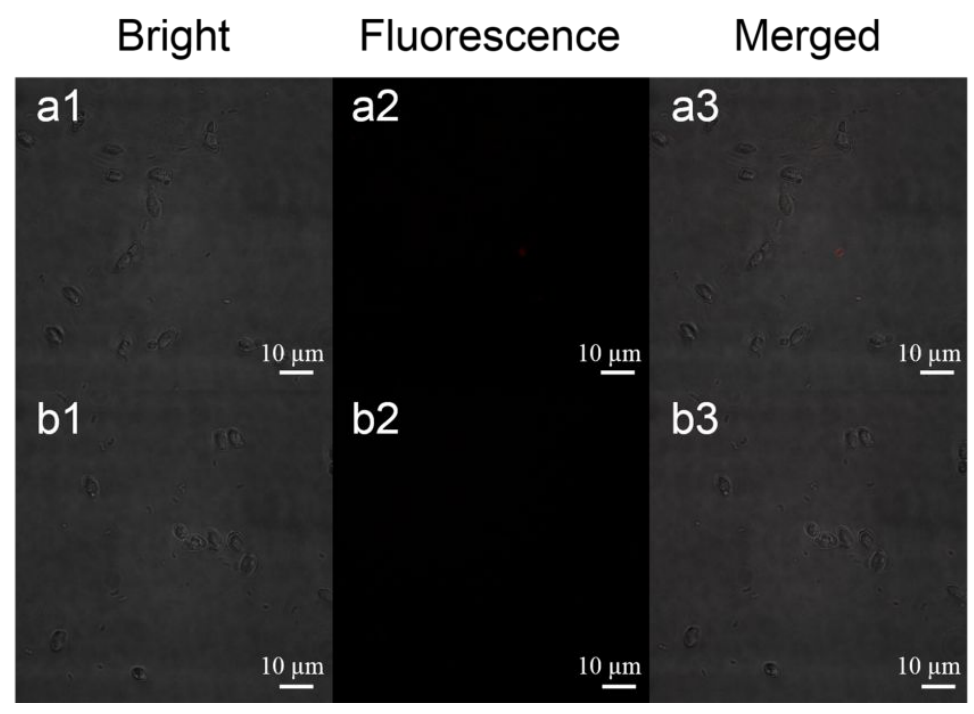

Figure S5. (a, a1, a2) CLSM images of Janus-poly(PEGDA)-cell without Bio-PEGACA after fluorescent staining. (b, b1, b2) CLSM images of Janus-poly(PEGDA)-cell without copolymerization of Bio-PEG-ACA with AAS after fluorescent staining. 